\title{
Multi-Trigger Resist for Electron Beam Lithography
}

\author{
Carmen Popescu, ${ }^{\mathrm{a}, \mathrm{b}}$ Alexandra McClelland, ${ }^{\mathrm{c}}$ Guy Dawson, ${ }^{\mathrm{b}} \mathrm{John}$ Roth, ${ }^{\mathrm{d}}$ Dimitrios Kazazis, ${ }^{\mathrm{e}}$ Yasin \\ Ekinci, ${ }^{\mathrm{e}}$ Wolfgang Theis, ${ }^{\mathrm{a}}$ Alex P.G. Robinson., ${ }^{\mathrm{b}, \mathrm{c}}$

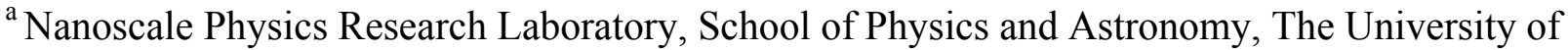 \\ Birmingham, Birmingham, B15 2TT, UK \\ ${ }^{\mathrm{b}}$ School of Chemical Engineering, University of Birmingham, Birmingham, B15 2TT, UK \\ ${ }^{\mathrm{c}}$ Irresistible Materials, Birmingham Research Park, Vincent Drive, Birmingham, UK \\ ${ }^{\mathrm{d}}$ Nano-C Inc., 33 Southwest Park, Westwood, MA 02090, USA \\ ${ }^{\mathrm{e}}$ Paul Scherrer Institute, 5232 Villigen, Switzerland \\ e-mail: a.p.g.robinson@bham.ac.uk
}

\begin{abstract}
Irresistible Materials is developing a new molecular resist system that demonstrates high-resolution capability based on the Multi-trigger concept. In a Multi-Trigger resist, multiple distinct chemical reactions in chemical amplification process must take place in close proximity simultaneously during resist exposure. Thus, at the edge of a pattern feature, where the density of photo-initiators that drive the chemical reactions is low, the amplification process ceases. This significantly reduces blurring effects and enables much improved resolution and line edge roughness while maintaining the sensitivity advantages of chemical amplification. A series of studies such as enhanced resist crosslinking, elimination of the nucleophilic quencher and the addition of high- $Z$ additives to e-beam resist (as a means to increase sensitivity and modify secondary electron blur) were conducted in order to optimize the performance of this material. The optimized conditions allowed patterning down to $28 \mathrm{~nm}$ pitch lines with a dose of $248 \mu \mathrm{C} / \mathrm{cm}^{2}$ using $100 \mathrm{kV}$ e-beam lithography, demonstrating the potential of the concept. Furthermore, it was possible to pattern $26 \mathrm{~nm}$ diameter pillars on a $60 \mathrm{~nm}$ pitch with dose of $221 \mu \mathrm{C} / \mathrm{cm}^{2}$ with a line edge roughness of $2.3 \mathrm{~nm}$.
\end{abstract}

Keywords: EUV lithography, molecular resist, multi-trigger resist, chemical amplification, resist sensitivity

\section{INTRODUCTION}

As the minimum lithographic feature size continues to shrink, the development of techniques and resist materials capable of high resolution (R), high sensitivity (S) and low line edge roughness (L) has become increasingly important for next generation lithography. However, the issue represents a fundamental trade-off in lithography (the RLS triangle) and it is difficult to overcome. In addition to fulfilling current resist targets for the next generation of devices, new material platforms must also have the potential to meet the outlined specifications beyond that point, to ensure a useful lifespan for next generation lithography. Traditional chemically amplified resist (CAR) materials have been extended to try and meet this need, but as resolution has improved, there have been significant decrease in sensitivity. [1,2] Addition of quenchers in a CAR reduces the acid diffusion length, and increases the resolution of the patterned features, but decreases the sensitivity, and impacts on material stochastics which leads to increase in line edge roughness. One current approach to boost the sensitivity in organic resists has been the addition of metals by incorporating organometallic complexes or metallic clusters into the resist, but again this can impact the line edge roughness. [3]

Additionally, as feature size requirements have progress below $20 \mathrm{~nm}$, pattern collapse upon development has become a serious limiting factor, independent of the lithography technique involved. At such small pitches the critical aspect ratio 
of collapse approaches 1:1 [4] requiring extremely thin resist films for successful patterning, which further increases line edge roughness and makes pattern transfer harder. One of the mechanisms involved in pattern collapse in high aspect ratio features is the poor adhesion between the resist material and the silicon substrate or underlayer. Therefore, alongside constantly developing and adapting resist platforms there is the need to introduce an interface that significantly improves the adhesion of the resist material to the silicon substrate, reducing pattern collapse and allowing for ultrahigh resolution, high aspect ratio patterning.

Irresistible Materials is developing a negative tone molecular resist platform, for electron beam lithography (e-beam) and extreme ultraviolet lithography (EUVL) applications. The resist demonstrates a good combination of photospeed, low line edge roughness (LER), and high-resolution patterning [5]. Existing state-of-the-art photoresists are based on the concept of chemical amplification, wherein each incoming photon causes multiple chemical reactions within the resist, significantly improving sensitivity. However, the amplification process is unconstrained and leads to blurring at the edge of features, reducing the achievable resolution. A variety of methods can be used to control the amplification, and improve resolution, such as the addition of base additives at low concentrations. However, these typically reduce the performance of the resist, for instance impacting on the dose, or increasing the LER because of material stochastics. [6] Irresistible Materials has developed a new category of resist chemistry that addresses these limitations called 'MultiTrigger Resist'. In a Multi-Trigger resist, multiple distinct chemical reactions must take place simultaneously and in close proximity for the amplification process to proceed. Thus, at the edge of a pattern feature, where the density of photo-initiators that drive the chemical reactions is low, the amplification process ceases. This significantly reduces blurring effects and enables much improved resolution and line edge roughness while maintaining the sensitivity advantages of chemical amplification.

In this study we report our ongoing efforts further improve the performance of this material. The molecular structure was modified to create enhanced versions of the standard resin that will offer higher cross-linking capability, greater molecular stiffness to reduce the LER, and ultimately higher resolution. The impact of metal additives was also evaluated. The resulting materials were patterned in e-beam lithography at $100 \mathrm{kV}$ and their lithographic properties were analyzed in comparison with our standard resist. To increase the adhesion of the resist to the wafer and reduce pattern collapse, a silane-based monolayer interface with crosslinking tail groups was investigated. Isolated pillar and semidense lines with aspect ratios above 1:1 were patterned in electron beam to evaluate the pattern collapse issue.

\section{EXPERIMENTAL}

The resist samples were prepared by dissolving the individual components in ethyl lactate. They were then combined in various weight ratios and concentrations. The resist was spun onto a proprietary carbon underlayer that we have developed consisting of a mixture in equal parts of a fullerene derivative and an epoxy crosslinker. Prior to the application of the underlayer the silicon substrates were cleaned in a three-step process: 10 min immersion in isopropyl alcohol (IPA) in an ultrasonic bath, 10 min immersion in piranha solution $\left(1: 1 \mathrm{H}_{2} \mathrm{SO}_{4}: \mathrm{H}_{2} \mathrm{O}_{2}\right)$ and finally 2 min dip in a weak aqueous solution of hydrofluoric acid (0.1-1\%).

After spin coating of the resist the samples received a post application bake. All 100kV e-beam exposures were performed using Vistec EBPG5000plus electron beam lithography system at the Paul Scherrer Institute, Switzerland. After exposure the samples were developed in n-butyl acetate for 60 seconds followed by an MIBC rinse.

Exposed samples were analyzed with a scanning electron microscope (SEM) in top-down view. Critical dimension (CD) and LER were calculated from the SEM images with the commercial software package SuMMIT. 

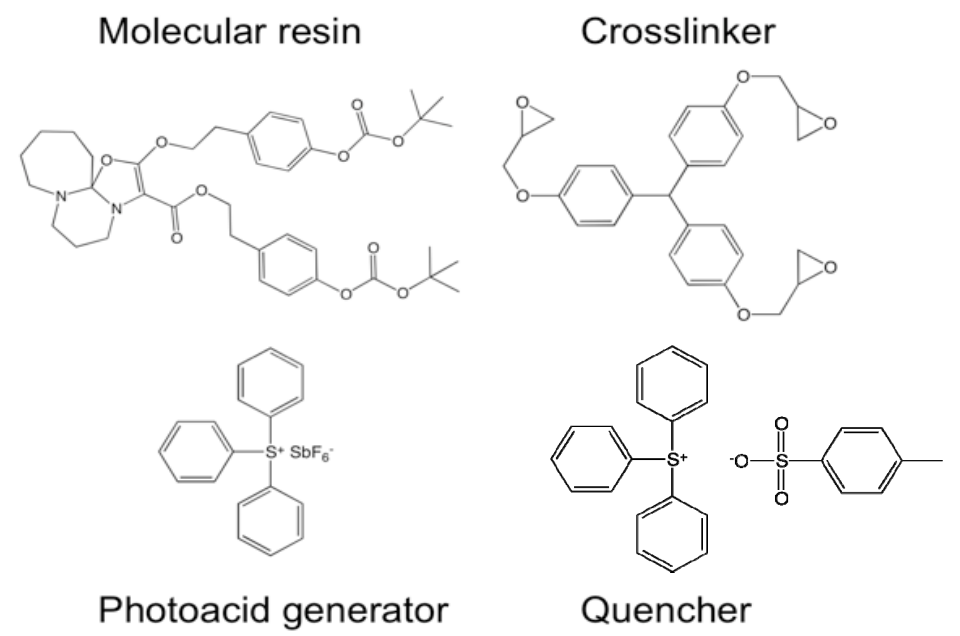

Figure 1: Baseline resist components of xMT resist

\section{RESULTS}

The baseline material for the optimization efforts is the previously introduced xMT resist [7]. It is a blended formulation of xMT resin, molecular epoxy crosslinker and a sulfonium photoacid generator mixed in 0.2:2:1 weight ratio with the addition of a quencher in the form of a photo-decomposable nucleophile that controls epoxy crosslinking $[8,9]$. The chemical structures of the components are shown in figure 1.

\subsection{Enhancement to base molecular resin}

We present enhancements to the base molecular resin itself (shown in Figure 1). One variant, EX2, is designed to stiffen the molecule to reduce line edge roughness, whilst another variant, EX3 also includes additional functional groups designed to increase sensitivity. The standard versions and EX3 version contain a quencher at $2.5 \%$ by weight. A quencher is often required in a Chemically Amplified Resist to try to reduce LER, at the expense of lower sensitivity. However, introducing a small amount of quencher into a system may introduce material stochastics, which will actually increase the LER, especially at the very high resolutions which are now required for the future lithography nodes. The EX2 version presented does not contain a quencher and thus relies on the Multi trigger effect described above for reducing blurring effects at the edge of the patterned feature.

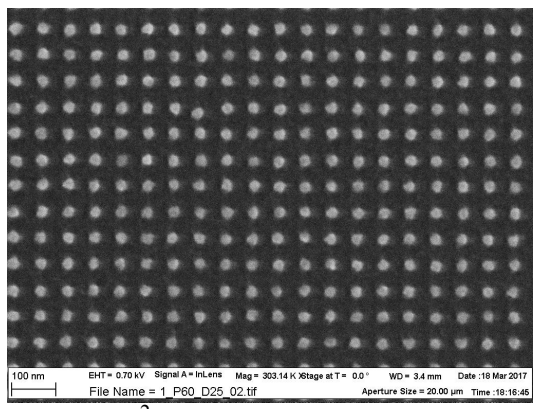

$407 \mu \mathrm{C} / \mathrm{cm}^{2}$, diameter $26.75 \mathrm{~nm}$ $\mathrm{LER}=2.14 \mathrm{~nm}$

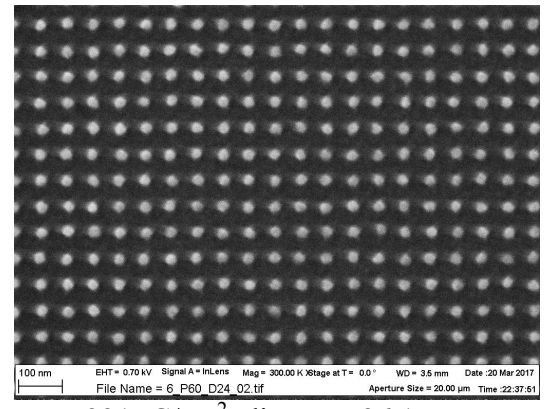

$221 \mu \mathrm{C} / \mathrm{cm}^{2}$, diameter $26.15 \mathrm{~nm}$ LER 2.32nm

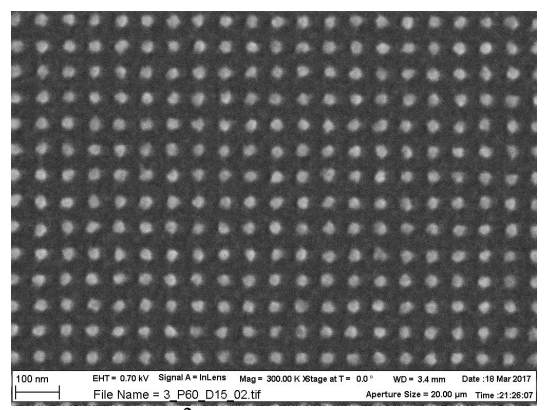

$303 \mu \mathrm{C} / \mathrm{cm}^{2}$, diameter $26.87 \mathrm{~nm}$ LER $3.04 \mathrm{~nm}$

Figure 2: SEM top down images of isolated pillars patterned in standard resin (left hand side), enhanced xMT version 2 - EX2 (middle) and enhanced xMT version 3 - EX3 (right hand side) 


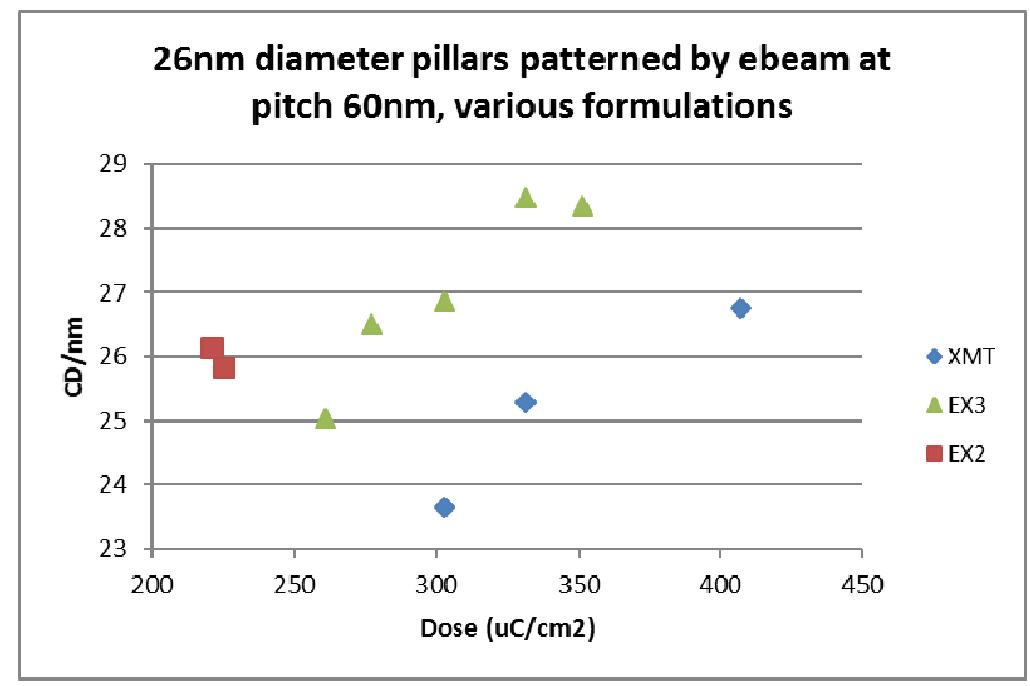

Figure 3: Graph showing pillar diameter variation with e-beam dose for different resist formulations

As can be seen from the results, the non-quencher version is significantly more sensitive, but has an LER approaching that seen in the standard version of the resist with quencher, offering an all-round better resist option.

\subsection{High-Z Additives}

Metal-based resists are currently receiving much attention, particularly for EUV lithography exposures. Metals often have a higher absorption at $13.5 \mathrm{~nm}$ wavelength radiation compared to organic resist materials, primarily due to their higher $\mathrm{Z}$ number [11]. Higher absorption may significantly improve sensitivity to enable high volume manufacturing. While many efforts focus on the higher absorption properties of metals, there is another aspect to high- $Z$ materials as well. Fundamental studies are currently under way about the different nature of light-resist interaction in the EUV range, but electron scattering properties are thought to be an important part of this. For example, they account for the secondary electron blur. Additionally, it is interesting to investigate whether the same effects are seen using e-beam lithography at different accelerating voltages. Therefore we have examined species of interest via the blending of high- $Z$ additives into the xMT resist, to create a hybrid resist. In a first instance, this concept is shown as a sensitizer to increase the sensitivity of the xMT but in further work we intend to screen for the differences in resist - electron interaction as well. So far we have tested about 20 different high-Z sensitizers, and seen a variety of different behaviours with the high- $Z$ addition both increasing or decreasing the sensitivity depending on the choice. The results below show one choice of sensitizer, patterned with $100 \mathrm{kV}$ ebeam lithography.
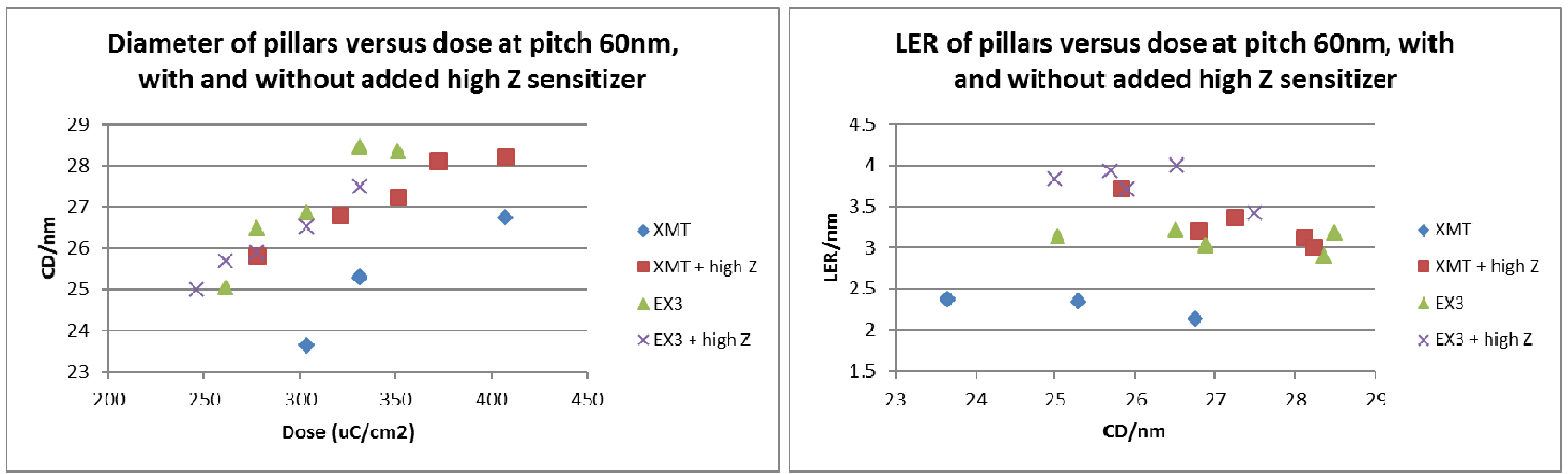

Figure 4: Changes in sensitivity and LER with added metal for pillars 

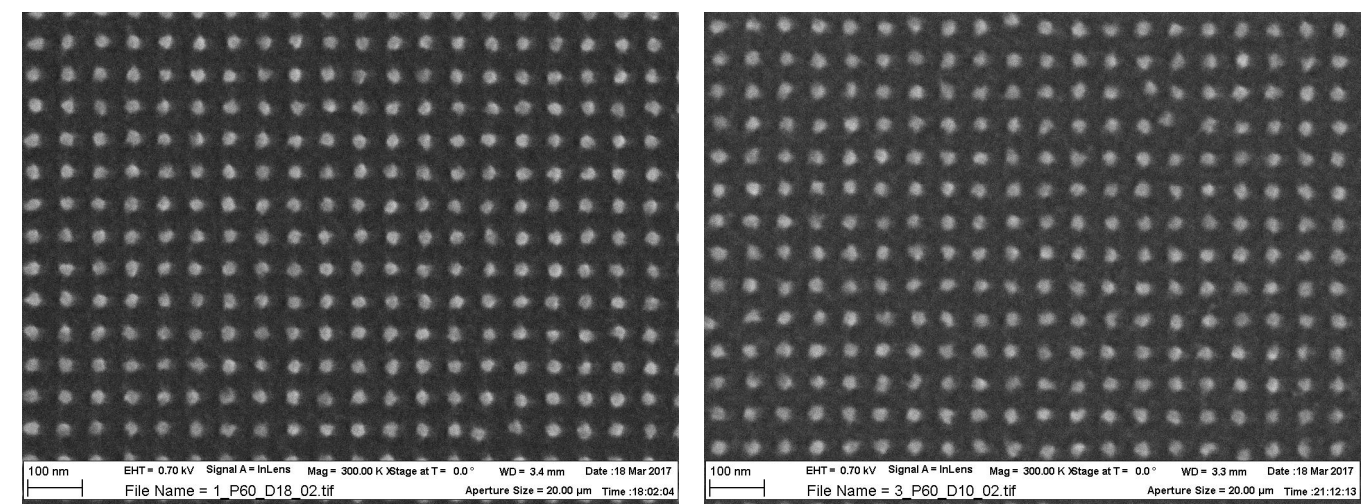

$331 \mu \mathrm{C} / \mathrm{cm}^{2}$, diameter $25.29 \mathrm{~nm}, 60 \mathrm{~nm}$ pitch, LER $2.35 \mathrm{~nm} 261 \mu \mathrm{C} / \mathrm{cm}^{2}$, diameter $25.04 \mathrm{~nm}, 60 \mathrm{~nm}$ pitch, LER $3.14 \mathrm{~nm}$

$\mathrm{xMT}+$ sensitizer

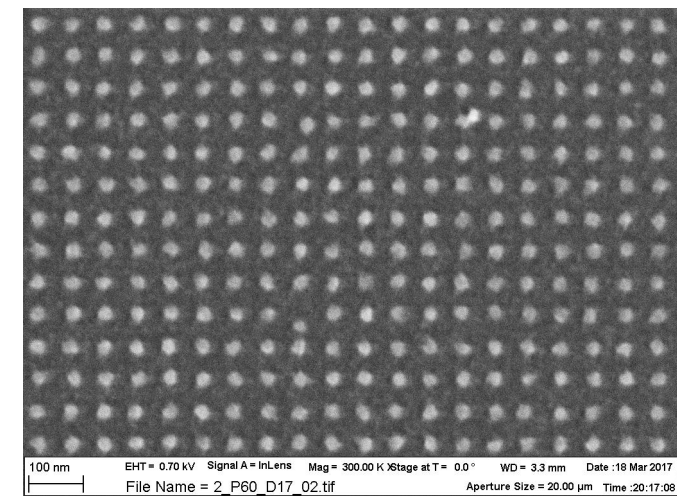

$\mathrm{EX} 3+$ sensitizer

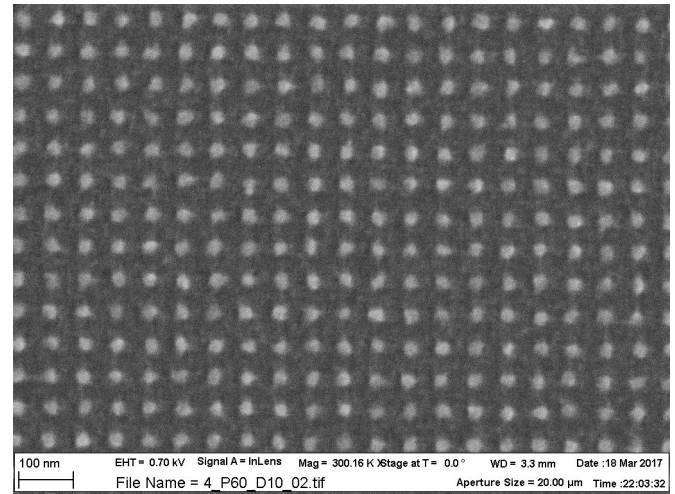

$\longmapsto \quad$ File Name $=4$ P P 60 D D 10_02.t

$321 \mu \mathrm{C} / \mathrm{cm}^{2}$, diameter $26.79 \mathrm{~nm}, 60 \mathrm{~nm}$ pitch, LER $3.21 \mathrm{~nm} 261 \mu \mathrm{C} / \mathrm{cm}^{2}$, diameter $25.69 \mathrm{~nm}, 60 \mathrm{~nm}$ pitch, LER $3.93 \mathrm{~nm}$

Figure 5: SEM top down images of isolated pillars patterned with added high Z sensitizer, xMT (left), and EX3 (right)

The results show that adding the high $\mathrm{Z}$ sensitizer increases the sensitivity of the resist. In the case of xMT, it decreases the dose required to get $26.0 \mathrm{~nm}$ pillar diameter by $25 \%$, from $375 \mu \mathrm{C} / \mathrm{cm}^{2}$ to $280 \mu \mathrm{C} / \mathrm{cm}^{2}$. For EX3, the improvement is less marked, from $287 \mu \mathrm{C} / \mathrm{cm}^{2}$ to $278 \mu \mathrm{C} / \mathrm{cm}^{2}$. The LER increases when the high-Z sensitizer is added. For $\mathrm{xMT}$, it increases from $2.2 \mathrm{~nm}$ to $3.6 \mathrm{~nm}$, and for EX3 it increases from $3.1 \mathrm{~nm}$ to $3.7 \mathrm{~nm}$. The standard deviation of the diameter of a set of pillars also increases when the high-Z sensitizer is added, from $1.0 \mathrm{~nm}$ to $1.5 \mathrm{~nm}$ for $\mathrm{xMT}$, and from $1.2 \mathrm{~nm}$ to $1.4 \mathrm{~nm}$ for EX3.

The same resists were used to pattern dense lines with 1:1 spacing. A similar trend was seen here as for the pillars, as shown in figure 6. The EX3 is faster than the xMT resist by $20 \%$. This is as expected due to the increased number of functional groups in EX3. Adding the high $\mathrm{Z}$ sensitizer decreases the dose to size by $18 \%$ for $\mathrm{xMT}$, and by a lesser amount, 13\%, for EX3. The LER of the lines increases when the high $\mathrm{Z}$ sensitizer is added, from $4.9 \mathrm{~nm}$ to $6.8 \mathrm{~nm}$ for xMT at $14 \mathrm{~nm}$ line width, and from $5.4 \mathrm{~nm}$ to $7.8 \mathrm{~nm}$ for EX3 at $14 \mathrm{~nm}$ line width. 

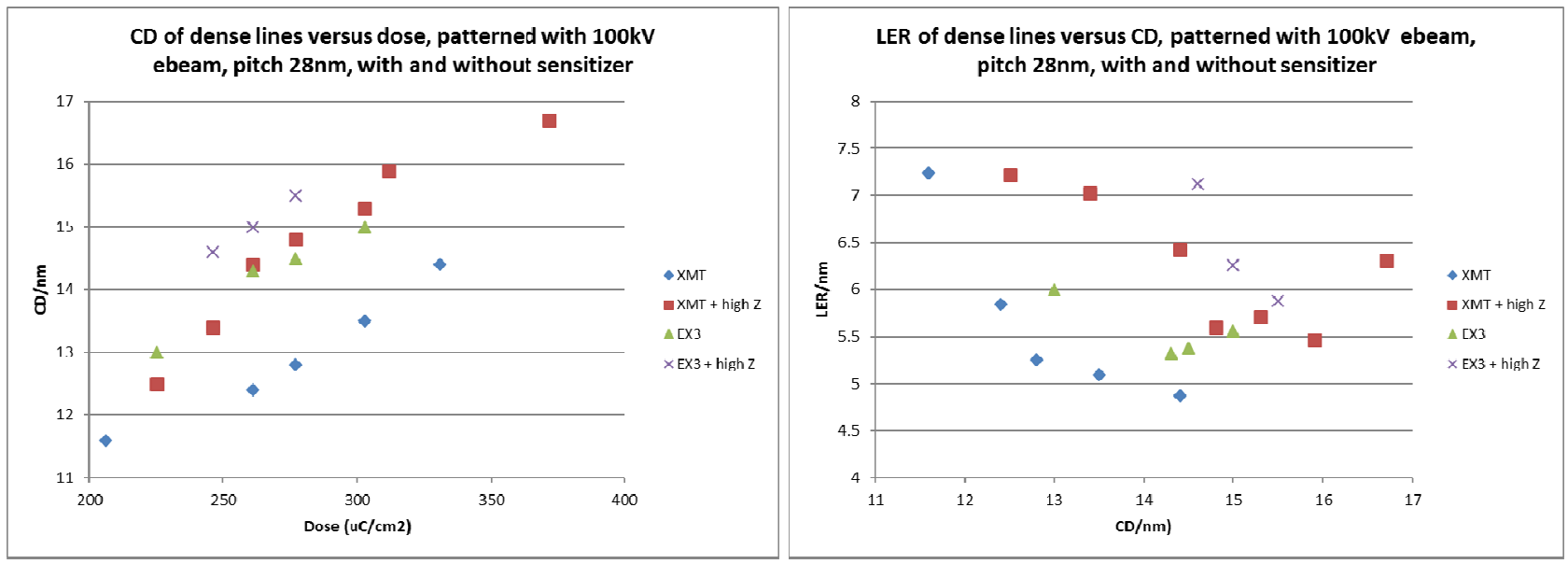

Figure 6: Changes in sensitivity and LER with added metal for dense lines

xMT

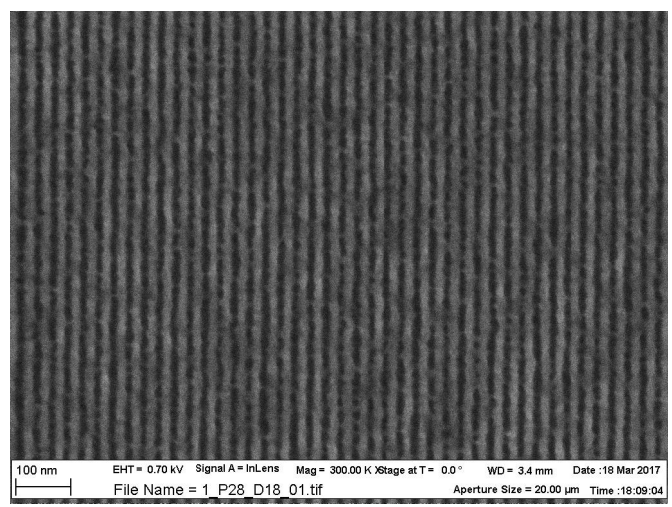

$331 \mu \mathrm{C} / \mathrm{cm}^{2}, \mathrm{CD} 14.4 \mathrm{~nm}$, pitch $28 \mathrm{~nm}$, LER $4.87 \mathrm{~nm}$

$\mathrm{xMT}+$ sensitizer

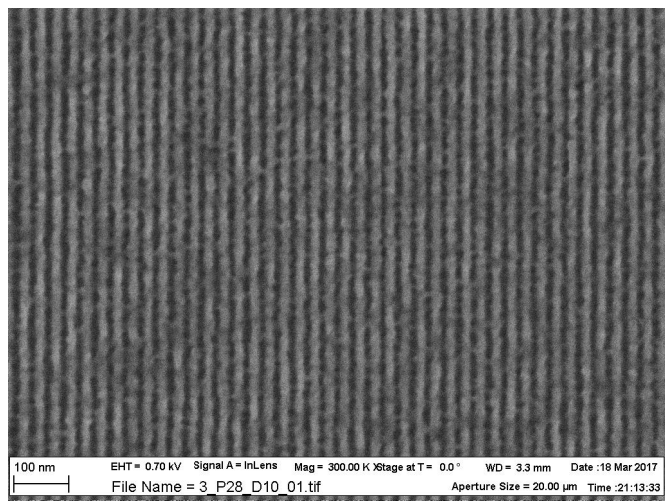

$261 \mu \mathrm{C} / \mathrm{cm}^{2}, \mathrm{CD} 14.4 \mathrm{~nm}$, pitch $28 \mathrm{~nm}$, LER $6.43 \mathrm{~nm}$
EX3

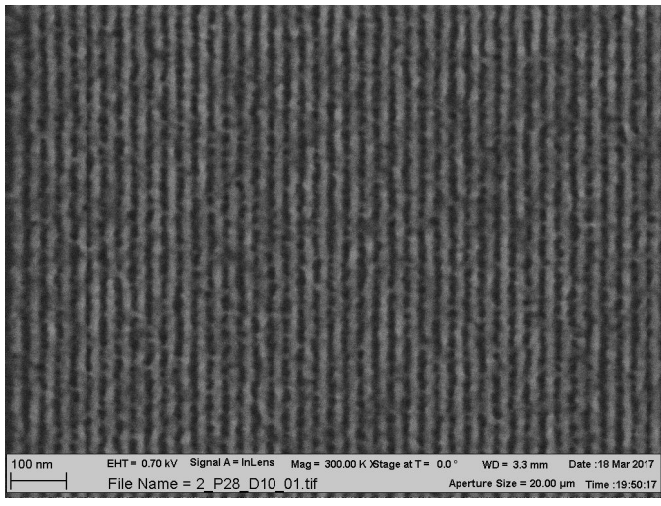

$261 \mu \mathrm{C} / \mathrm{cm}^{2}, \mathrm{CD} 14.3 \mathrm{~nm}$, pitch $28 \mathrm{~nm}$, LER $5.32 \mathrm{~nm}$

$\mathrm{EX} 3+$ sensitizer

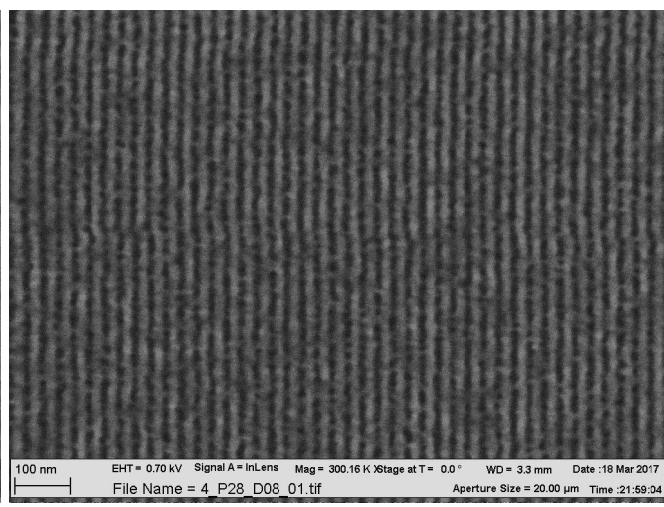

$261 \mu \mathrm{C} / \mathrm{cm}^{2}$, CD $14.6 \mathrm{~nm}$, pitch $28 \mathrm{~nm}$, LER $7.12 \mathrm{~nm}$

Figure 7: SEM top down images of dense patterned with added high Z sensitizer, xMT (left), and EX3 (right) 


\subsection{Silane-based monolayer interface}

We have investigated the effect of introducing a silane based monolayer interface between the organic resist material and the silicon wafer. The interface monolayer contains molecules, which have dual chemical functionality (organic and inorganic). A central silicon atom gives the molecule reactivity towards the silicon substrate, and an organic functional group attached to silicon atom offers organic reactivity towards the resist. The inorganic entities condense with themselves to give an oligomeric structure and offer integrity and stability to the structure through chemical bonds between the organic and inorganic surfaces promoting the adhesion between these two dissimilar materials. A schematic of the entire process from surface modification to lithography and development is shown in figure 8.

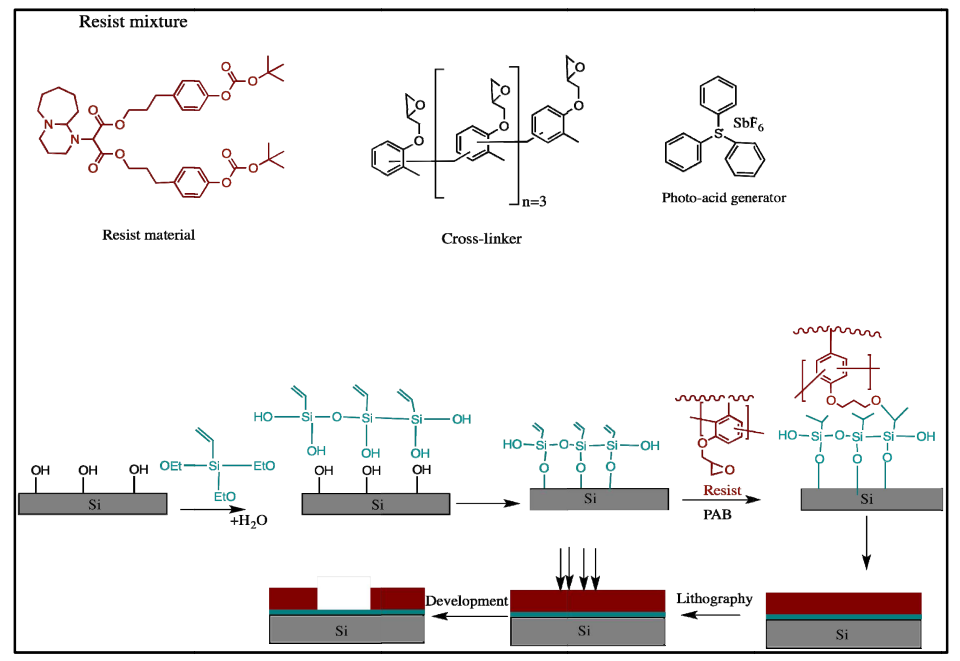

Figure 8: Schematic of the resist processing showing the surface hydroxylation, surface silanization, resist deposition, lithography patterning and development

Dense lines at $18 \mathrm{~nm}$ half pitch were patterned on a thicker xMT resist film on the silane-based underlayer in order to investigate the pattern collapse issue. Figure 9 shown bellow demonstrates the collapse occurring in the first sample (left), which is spun on the standard carbon underlayer. The pattern collapse is mitigated at the introduction of the silane interface (right image in Figure 9).

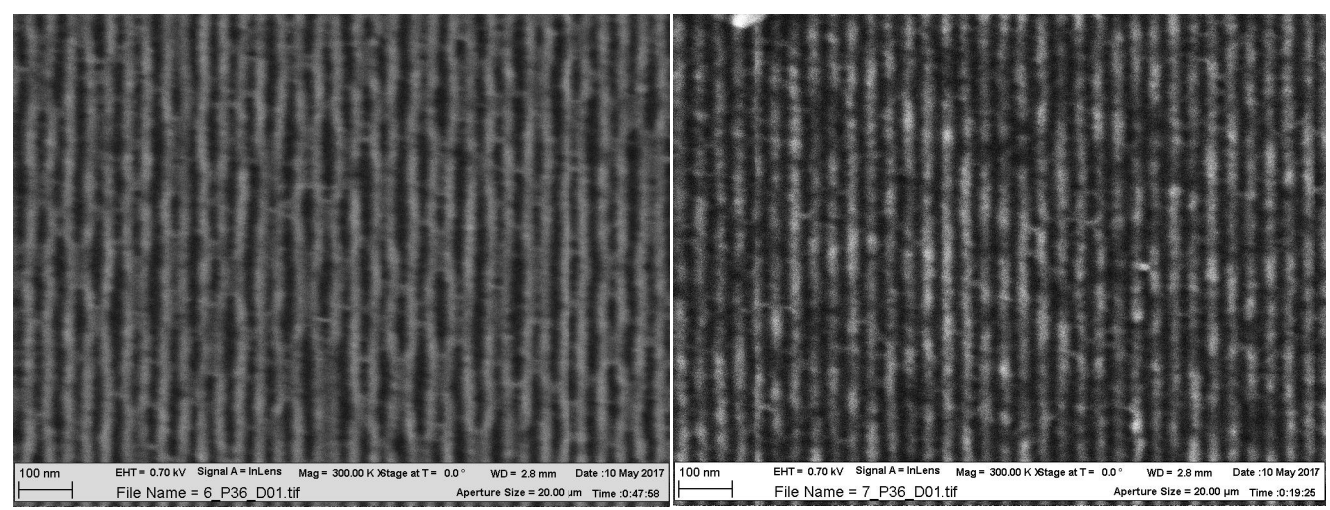

Figure 9: SEM top down images of dense pattern of xMT on standard carbon underlayer (left) and silane-based underlayer (right)

Isolated pillars $(25 \mathrm{~nm}$ diameter) were also patterned on the above-described adhesion promiting layer. Due to the increase in the degree of freedom in the pillars pattern, the pattern collapse becomes even more likely. Figure 10 shows a comparison between the pillars on standard carbon underlayer and the silane-based underlayer. Pattern collapse is present on both samples with the difference that the features on the silane underlayer show a preferred direction of collapsing. This may be due to the increased mechanical strength of the features at the interface with the wafer. More investigation needs to be undertaken to understand and improve the collapse mechanism in pillars. 

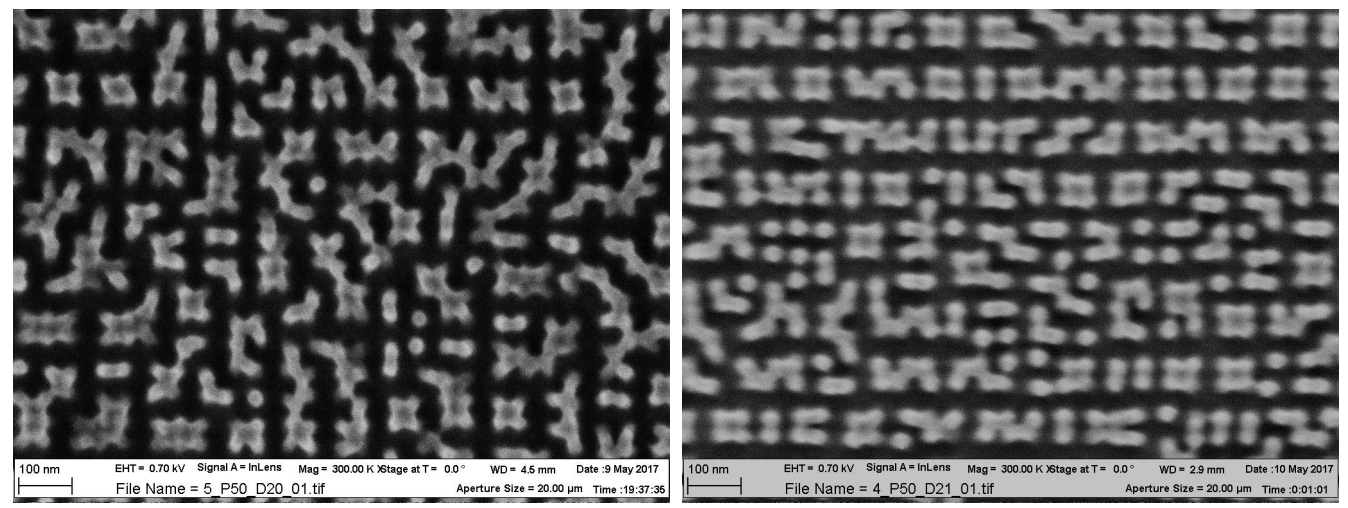

Figure 10: SEM top down images of isolated pillars of xMT on standard carbon underlayer (left) and silane-based underlayer (right)

\section{CONCLUSION}

We have undertaken a series of studies to optimize the performance a negative tone molecular resist platform. These include enhanced resist crosslinking, elimination of the nucleophilic quencher and the addition of high-Z additives to ebeam resist (as a means to increase sensitivity and modify secondary electron blur) were conducted in order to optimize the performance of this material. The optimized conditions allowed patterning $14 \mathrm{~nm}$ half-pitch (hp) lines with a dose of $248 \mu \mathrm{C} / \mathrm{cm}^{2}$ using $100 \mathrm{kV}$ ebeam lithography. Furthermore it was possible to pattern $26 \mathrm{~nm}$ diameter pillars on a $60 \mathrm{~nm}$ pitch with dose of $221 \mu \mathrm{C} / \mathrm{cm}^{2}$ with an LER of $2.3 \mathrm{~nm}$. The introduction of a silane-based interface between the resist film and the wafer showed the mitigation of pattern collapse in $18 \mathrm{~nm}$ dense lines at aspect ratio above 1:1. Future work will focus on driving the resist more fully towards Multi-Trigger behavior, e.g. without added quencher, and using alternative crosslinkers for more selective crosslinking to improve LER and resolution.

\section{ACKNOWLEDGEMENTS}

The authors would like to thank the Engineering and Physical Sciences Research Council (EPSRC) and the Nanoscience Foundries and Fine Analysis for support of this project. This project has received funding from the EU-H2020 research and innovation programme under grant agreement No 654360 having benefitted from the access provided by Paul Scherrer Institute in Villigen, Switzerland within the framework of the NFFA-Europe Translational Access Activity. The authors thank Irresistible Materials Ltd. and Nano-C for support and provision of resist materials. The Disco DAD 321 wafer dicer used in this research was obtained through the Birmingham Science City Creating and Characterizing Next Generation Advanced Materials, with support from Advantage West Midlands (AWM) and part funded by the European Regional Development Fund (ERDF). C.P. thanks the University of Birmingham for support. C.P. and A.P.G.R. gratefully acknowledge the support and assistance of Professor R E Palmer.

\section{REFERENCES}

[1] Fujii, T., Matsumaru, S., Yamada, T., Komuro, Y., Kawana, D., Ohmori, K., "Patterning performance of chemically amplified resist in EUV lithography," Proc SPIE 9776, 97760Y (2016).

[2] Tsubaki, H., Nihasi, W., Tsuchihashi, T., Yamamoto, K., Goto, T., "Negative-tone imaging with EUV exposure toward $13 \mathrm{~nm}$ hp,” Proc SPIE 9776, 977608 (2016).

[3] Stowers, J., Anderson, J., Cardineau, B., Clark, B., De Schepper, P., Edson, J., Greer, M., Jiang, K., Kocsis, M., Meyers, S., Telecky, A., Grenville, A., De Simone, D., Gillijns, W., Vandeberghe, G., "Metal oxide EUV photoresist performance for N7 relevant patterns and processes," Proc SPIE 9779, 977904 (2016).

[4] Hien, S., Rich, G., Molina, G., Cao, H. B., Nealey, P. F., "Collapse behavior of single layer 193 and 157 nm resists: Use of surfactants in the rinse to realize the sub $130 \mathrm{~nm}$ nodes," Proc SPIE 4690, 254-261 (2002). 
[5] Frommhold, A., McClelland, A., Roth, J., Fallica, R., Ekinici, Y., Robinson, A. P. G., "Optimization and sensitivity enhancement of high-resolution molecular resist for EUV lithography," Proc. SPIE 9776, 977614 (2016).

[6] Cardineau B, Early W, Fujisawa T, Maruyama K, Shimizu M, Sharma S, Petrillo K, Brainard R., "LER limitations of resist thin films," J Photopolym Sci Technol 25, 633-40 (2012).

[7] Frommhold, A., McClelland, A., Yang, D. X., Palmer, R. E., Roth, J., Ekinci, Y., Rosamund, M. C., Robinson, A. P. G., "Towards $11 \mathrm{~nm}$ half-pitch resolution for a negative-tone chemically amplified molecular resist platform for extreme-ultraviolet lithography," Proc. SPIE 9425, 942504 (2015).

[8] Lawson, R. A., Chun, J. S., Neisser, M., Tolbert, L. M., Henderson, C. L., "Methods of controlling cross-linking in negative-tone resists," Proc. SPIE 9051, 90510Q (2014).

[9] Frommhold, A., Yang, D. X., McClelland, A., Xue, X., Ekinci, Y., Palmer, R. E., Robinson, A. P. G., "Optimization of Fullerene-based Negative tone Chemically Amplified Fullerene Resist for Extreme Ultraviolet Lithography," Proc. SPIE 9051, 905119 (2014).

[10] Fallica, R. A., Stowers, J. K., Grenville, A., Frommhold, A., Robinson, A. P. G., Ekinci, Y., "Dynamic absorption coefficients of CAR and non-CAR resists at EUV,” Proc. SPIE 9776, 977612, (2016). 